\title{
Narración subjetiva: Una aproximación conceptual al relato de vida en el contexto de la educación artística' ${ }^{1}$
}

\author{
Subjective Narrative: A Conceptual Approach to the Life History Method in the Context \\ of Visual Arts Education ${ }^{2}$
}

\section{Narração subjetiva: uma aproximação conceitual ao relato de vida no contexto da educação artística visual ${ }^{3}$}

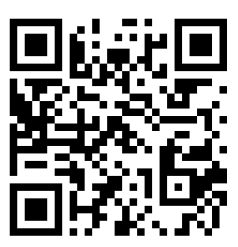

\author{
Patricia Raquimán-Ortega \\ Universidad Metropolitana de Ciencias de la Educación \\ Santiago, Chile \\ patricia.raquiman@umce.cl \\ (iD) https://orcid.org/0000-0003-0139-7516 \\ Miguel Zamorano-Sanhueza \\ Universidad Metropolitana de Ciencias de la Educación \\ Santiago, Chile \\ miguel.zamorano@umce.cl \\ iD https://orcid.org/0000-0002-8136-7001
}

\begin{abstract}
Recibido • Received • Recebido: 03 / 12 / 2018
Corregido • Revised • Revisado: 22 / 05 / 2020

Aceptado $\cdot$ Accepted $\cdot$ Aprovado: $18 / 08 / 2020$
\end{abstract}

\begin{abstract}
Resumen: Este artículo explora un marco conceptual que rescata los relatos de vida como un enfoque para investigar algunas ideas en torno a la enseñanza de las artes visuales. Tiene como perspectiva expandir la noción del proceso de enseñanza y aprendizaje, ligándolo al entorno vital, para destacar la percepción de lo cotidiano. Con esto, las ideas desarrolladas pretenden generar una reflexión acerca de cómo las percepciones subjetivas del contexto en que se desenvuelven el profesorado y estudiantado se transforman en una oportunidad para la comprensión del espacio cercano y sus relaciones. La narración, sustentada en historias de vida, permite tener cercanía con sus modos de reconstruir la experiencia en la enseñanza de las artes visuales.
\end{abstract}

Palabras claves: Historia de vida profesional; artes visuales; educación artística; investigación de las artes visuales.

\footnotetext{
1 Este proyecto es parte del proyecto de investigación es FGI 38-18 "Decolonizar el territorio: En búsqueda de historias de vida y bitácoras visuales de profesores (as) en ejercicio en la enseñanza de las Artes Visuales". Financiado por la Dirección de Investigación de la Universidad Metropolitana de Ciencias de la Educación de la Educación, Santiago, Chile.

${ }^{2}$ This project is part of the research project titled FGI 38-18“Decolonizing the territory: in search of life stories and visual logs of teachers in the teaching of the Visual Arts", which is funded by the Research Direction of the Universidad Metropolitana de Ciencias de la Educación, Santiago, Chile..

${ }^{3}$ Este projeto faz parte do projeto de pesquisa FGI 38-18“Descolonizar o território: em busca de histórias de vida e diários visuais de professores em exercício no ensino das Artes Visuais". Financiado pela Direção de Investigação da Universidade Metropolitana de Ciências da Educação, Santiago, Chile.
} 
http://doi.org/10.15359/ree.24-3.29

http://www.una.ac.cr/educare

educare@una.ac.cr

\begin{abstract}
This article explores a conceptual framework that considers life histories as an approach to examining some ideas about Visual Arts teaching. This essay tries to expand the notion of the teaching and learning process, linking it to the vital environment to highlight daily life interpretation. From this point, this essay reflects on how the context in which teachers and students live becomes an opportunity to understand the near space and its relationships. With the support of life histories, narratives allow for closeness to their ways of reconstructing the experience in teaching the visual arts.
\end{abstract}

Keywords: Professional life history; visual arts; artistic education; visual arts research.

Resumo: Este artigo explora uma estrutura conceitual que resgata histórias de vida como uma abordagem para investigar algumas idéias sobre o ensino de artes visuais. Este ensaio tem como perspectiva ampliar a noção de processo de ensino e aprendizagem, ligando-o ao ambiente de vida, para destacar a percepção do cotidiano. Com isso, o estudo objetiva refletir sobre como o contexto em que professores e alunos se desenvolvem torna-se uma oportunidade para compreender o espaço próximo e suas relações. A narração sustentada em histórias de vida permite ter proximidade com seus modos de reconstruir a experiência no ensino das artes visuais.

Palavras-chave: História da vida profissional; artes visuais; educação artística; pesquisa em artes visuais.

\title{
Acercamiento de conceptos
}

La investigación en educación artística se vale de diferentes métodos, unos más convencionales o novedosos que otros, para comprender de qué modo la enseñanza y el aprendizaje de las artes visuales se manifiestan en el espacio del aula. Para ello, es relevante considerar las apreciaciones y los discursos declarados por diferentes docentes. En estos discursos, emitidos desde la demarcación de una experiencia, aquellas metodologías que destacan la subjetividad de los enunciados en ciertos contextos educativos pueden dar lugar a otras modalidades de comprensión. Dentro de las formas menos tradicionales de indagación, las metodologías cualitativas constituyen, ya desde los inicios del siglo XX, posibilidades teóricas concretas de entrar en la comprensión de una complejidad dada en las percepciones individuales de los sujetos. Dentro de estas metodologías, los modos de investigación basados en las narrativas parecen vincularse, de manera muy consistente, con los procesos de creación artística y -tal como lo postula Agra Pardiñas (2006) - con el proceso mismo de aprendizaje artístico: "la narrativa, como el arte, responde a una cualidad esencialmente humana, es sensible a los valores del contexto contemporáneo, propone un modo cualitativo de conocer y es una forma de expresión artística" (p.134). Se entiende que la narrativa personal se encuentra inscrita dentro de formas de representación que son alternativas al discurso verbal y que incluyen otras formas de "escritura del yo" dadas como manifestaciones artísticas (Agra Pardiñas, 2006, p. 135).

Para Weber (2001), la investigación sociológica está basada en el hecho de que los actores sociales utilizan parámetros subjetivos. Al estudiar estas relaciones, es posible constatar que se encuentran compuestas por acciones relacionadas unas con otras, las cuales pueden ser

2

Patricia Raquimán-Ortega y Miguel Zamorano-Sanhueza

Los artículos de la Revista Electrónica Educare del Centro de Investigación y Docencia en Educación de la Universidad Nacional, Costa Rica, se comparten bajo términos de la Licencia Creative Commons: Reconocimiento, No Comercial, Sin Obra Derivada 3.0 Costa Rica. Las autorizaciones adicionales a las aquí delimitadas se pueden obtener en el correo: educare@una.cr 
http://doi.org/10.15359/ree.24-3.29

comprendidas como fenómenos que poseen un significado concreto. Esta perspectiva permite considerar un punto de vista diferente sobre la educación artística y sus modos de investigación. La posición weberiana se basa en que "la ciencia no puede entregar conocimientos sobre la realidad que van más allá de nuestras vivencias acerca de ella" (Mella Valenzuela, 2003, p. 59); por ello, analizar las vivencias de los actores permite tener en cuenta las posibles relaciones y asociaciones entre los diferentes elementos que componen sus historias. Situarse desde un paradigma comprensivo para entender la realidad es reconocer la experiencia vivida y narrada como un desafío para interpretar la vida de otro sujeto. Será una acción que requiere examinar aspectos éticos y una preparación teóricoconceptual por parte de quien investiga, de manera que observe variables de tipo cualitativo.

Retomando las palabras de Weber (2001), la acción humana puede explicarse, a partir del enfoque comprensivo, como una postura que adquiere el sujeto investigador al sopesar el sentido de la acción social. Esta operación, entendida como interaccionismo simbólico (de aquí en adelante, IS), proporciona un cuadro coherente acerca de la naturaleza de esta acción de tipo colectivo y toma en cuenta la necesidad de tener acceso al conocimiento de la vida de los individuos. El IS considera que los significados son productos sociales formados por las actividades que las personas realizan y su interacción (Blumer, 1998). El IS descansa en las siguientes premisas: Los seres humanos actúan hacia las cosas sobre la base de los significados que estas tienen para ellos; el significado de tales cosas se deriva o surge de la interacción social que tiene con los demás individuos; estos significados se manejan y modifican por medio de un proceso de interpretación que utilizan las personas al tratar con las cosas que encuentran (Blumer, citado en Schwartz y Jacobs, 1984).

A partir de este marco conceptual, este texto plantea una reflexión acerca de la posición del propio profesor y profesora como núcleos de enunciación fundados en sus propias historias de vida. Si las acciones son forjadas por la persona actora desde lo que percibe e interpreta, es necesario ver y percibir la situación tal como el profesorado la observa, determinar su relevevancia en términos del significado que tienen para él. Por este motivo, su relato de vida profesional se constituye como un eje articulador, una narrativa comprendida como "la cualidad estructurada de la experiencia entendida y vista como un relato" (Bolívar Botía, 2002, p. 5). Este relato posee"pautas y formas de construir sentido, a partir de [actos] temporales personales, por medio de la descripción y el análisis de los datos biográficos" (Bolívar Botía, 2002, p. 5). Una narración permite la comprensión de la complejidad de lo relatado por los individuos, donde aparecen conflictos y dilemas presentes en sus vidas.

Entre tantas funciones que asume en la vida teórica y práctica, la narración es principalmente un método (un camino = odos) ancho y común para acceder a la realidad de algo. Un camino que en vez de subsumir lo desconocido presente bajo una ley conocida, como hacen las ciencias en general, da cuenta de, o mejor, cuenta, cómo tal cosa determinada ha llegado a ser lo que es, a través de una historia también determinada. (Giannini, 2004, p. 86) 
http://doi.org/10.15359/ree.24-3.29

http://www.una.ac.cr/educare

educare@una.ac.cr

La narración permite abrir nuevas posibilidades de comprensión, para así valorar la riqueza de las experiencias, al vincular el conocimiento que nace de la propia acción. Las historias de vida permiten acceder a la cotidianeidad, a las vivencias en contextos institucionales determinados, captando la voz de los actores sociales y convirtiéndolos en el centro de la construcción interpretativa. Esta ópción permite considerar que "la narrativa no sólo expresa importantes dimensiones de la experiencia vivida, sino que, más radicalmente, media la propia experiencia y configura la construcción social de la realidad" (Bolívar Botía et al., 2002, p. 4).

La articulación de una biografía sustentada en el relato no se hace en el vacío, hay una verdadera construcción que se levanta para tomar la coherencia entre las contradicciones de lo vivido, la mirada subjetiva desde la experiencia y articularlo con el contexto social (de Villers, 1996). La narración de un individuo es más que una historia singular, ella podría constituirse como un relato colectivo, en el cual puede encontrarse manifestada toda una sociedad. Para ello, considerar las historias de un grupo de individuos sobre un acontecimiento en particular entrega una mirada colectiva de los acontecimientos que proporcionan una mayor densidad de datos en función de una comprensión profunda.

Se pueden establecer diferentes vías de comunicación entre "el punto de vista exterior del observador y las formas en que los actores perciben y viven lo que hacen mientras actúan" (Corcuff, 1998, p. 15). La persona investigadora debe integrar la reflexión sobre las acciones de los actores individuales, "de nada sirve leer los grandes procesos sociales si se es incapaz de comprender la vida de las personas: la forma en que viven, luchan y afrontan el mundo" (Martuccelli y de Singly, 2012, p. 11). En este contexto, la experiencia personal del individuo se explica en la intersubjetividad social (Albero et al., 2008), el énfasis se dará en la necesidad de interpretar qué está pasando en la sociedad percibida como un todo y cómo se va accediendo al significado que tienen para sus participantes.

Por último, desde esta perspectiva, se llega al entendimiento interpretativo de los datos, como un medio para develar la acción social en la cual está inserto el individuo. Entender el significado es central, para ello es relevante verificar la interpretación subjetiva, por medio de la comparación, con el curso concreto de los acontecimientos. Los relatos, en cuanto a su papel de eje articulador, expresan la conciencia que tienen los sujetos de sus propios procesos de cambio en su vida profesional. Los relatos entregan un recorrido de los hechos tal como fueron ocurriendo, no son solo "fruto de un registro objetivo de los hechos, sino una construcción para dar significado a la propia vida" (Bolívar Botía et al., 2001, p. 141), sino que plantean el desafío de comprender cómo cobraron sentido, lo cual permite visualizar diferentes estrategias en las prácticas pedagógicas individuales. 


\section{La biografía como un método investigativo}

Los relatos de vida se hacen parte del método biográfico, esto es, un "conjunto de técnicas metodológicas basadas en la [búsqueda abierta] sobre las historias de vida, ... relatadas por los propios sujetos" (Kornblit, 2004, p. 15). Esta perspectiva se ha desarrollado como una búsqueda de significado, otorgado por los mismos individuos a diversos acontecimientos vitales de sus historias de vida. Así, "para los científicos sociales, las experiencias particulares de las personas recogidas a través de las historias de vida representan la posibilidad de recuperar los sentidos, vinculados con las experiencias vividas" (Kornblit, 2004, p. 15). Esta perspectiva entrega la posibilidad de encontrar connotaciones que, en el plano de lo íntimo, plantean el desafío de comprender el contexto social en el que surgen, intentando trascender desde lo particular hacia una construcción de un saber propiamente social.

Esta mirada del conocimiento social a partir de la subjetividad permite considerar los relatos como una expresión de importantes dimensiones acerca de experiencias vividas que configuran la construcción social de la realidad. En las ciencias sociales, el reconocimiento de la historia de un individuo, como una manera de comprender la realidad, tuvo largos tiempos de espera para ser reconocida. Al respecto, cabe mencionar las primeras obras que incursionaron en las historias de vida, la obra pionera de Thomas y Znaniecki (2004): El campesino polaco en Europa y América (1918-1920), la cual recolectó más de setecientas cartas y un relato de vida en profundidad de inmigrantes de Polonia que residían en Estados Unidos. Las investigaciones se limitan a recogerlo debidamente ordenado y transcrito, con un prólogo, sin someterlo a ningún tipo de análisis.

Otra obra representativa es el trabajo de Lewis (1964): Los hijos de Sánchez, la que desarrolla biografías sobre la cultura de la pobreza. De extrema importancia es también La miseria del mundo, de Bourdieu (1999), cuyo objetivo fue entregar una mirada comprensiva sobre los nuevos problemas sociales que emergieron en la Francia de la década de los 90. El texto aborda testimonios de hombres y mujeres que, de manera libre, relataron diferentes aspectos de sus vidas. En este se tomó en cuenta el ámbito socio económico donde se encontraban inscritos, y en muchas ocasiones no resultaron fáciles de abordar.

Estos trabajos visionarios consideran al ser humano y sus relatos como evidencias en la comprensión de fenómenos sociales. Al mismo tiempo, se desea validar al sujeto como poseedor de respuestas que pueden llegar a ayudar a la comprensión de la realidad. Lo que caracteriza a los seres humanos es la capacidad de entenderse a través de la conversación, la cual "se centra en la exploración hermenéutica del ser histórico tal como se expresa en la tradición del lenguaje", según Gadamer (citado en Freire Hermosilla et al., 2005, p. 56).

La expresión "relato de vida" se introdujo en Francia aproximadamente en los años 50 (Bertaux, 1997), hasta ese entonces se utilizaba el término de "historia de vida", pero este 
http://doi.org/10.15359/ree.24-3.29

http://www.una.ac.cr/educare

educare@una.ac.cr

concepto tenía el inconveniente de no distinguir entre la historia vivida por una persona y el relato que ella podría hacer de su historia, a petición de una investigadora o un investigador, en un momento determinado de su vida (Bertaux, 2005). El recurso de los relatos de vida ha permitido enriquecer una dimensión diacrónica de la subjetividad y una configuración de las relaciones sociales en su desarrollo histórico, situado de manera específica.

La oportunidad que brinda el utilizar los relatos de vida es la de circunscribirse a la comprensión de los fenómenos sociales desde la complejidad y la subjetividad que proporciona la interpretación personal de las vivencias en diferentes dominios que van desde lo personal hasta lo profesional. Al trazar relaciones entre las prácticas pedagógicas, mediante los relatos que el profesorado hace en torno a su percepción de los hechos y fenómenos vinculados a su práctica, relacionando esta mirada con su desempeño en la enseñanza de las artes visuales, es posible obtener una visión de mayor densidad con respecto a su acción docente.

Los relatos de vida, al resaltar las experiencias vitales de los individuos en su acción cotidiana, relevan tanto el peso de las vivencias individuales en los marcos institucionales en que se desenvuelven, como también el impacto de las decisiones personales en los procesos de cambio y estructuración social (Aceves Lozano, 1999).

La tendencia más común para comprender las acciones que el profesorado realiza en sus prácticas es tomar en cuenta análisis que se sitúan externamente al mundo cotidiano de la práctica de la profesora o profesor en ejercicio. Debido a ello, el enfoque sobre los relatos de vida se convierte en la opción más pertinente para recuperar aquella subjetividad que es testigo de los desafíos dados en la enseñanza de las artes visuales, ya que la teorización externa a los sujetos de estudio se reemplaza por la preeminencia de los propios relatos conscientes de su protagonismo en la experiencia vivida, "la complejidad de la realidad contenida bajo la denominación de lo artístico requiere de múltiples formas de aproximación"(Luque Ramosy Cruz Elviara, 2016, p. 44), y la aproximación de las historias de vida permite una mejor comprensión de lo social y lo artístico, de un modo mucho más integral.

\section{La verdad narrativa y sus desafíos}

El relato de vida constituye una verbalización que desea rescatar el significado de la propia vida profesional de cada profesora o profesor. En un relato de este tipo hay una recreación personal cuya complejidad no es transferible, y si bien el recuerdo puede no ser exacto con respecto a los acontecimientos mismos, sí resulta ser significativo en el presente, como consolidación comprensiva de aquello que se considera importante. Aquello que se cuenta va acompañado de una interpretación: "en una auto biografía la verdad es la forma en que el/la protagonista interpreta lo sucedido" (de Miguel 1996, en Bolívar Botía et al., 2001, p. 142). 
El foco de este tipo de indagación es establecer la narración como una visión subjetiva de la realidad, ya que se espera que un relato nunca sea un registro de lo que ha sucedido como una bitácora rigurosa de acontecimientos, sino que, muy por el contrario, se trata de una interpretación de la experiencia del individuo, "cuando el conocimiento es representado como un collage, o una red o entramado interconectado, la investigación es menos propensa a proceder de modo jerárquico, siguiendo la lógica tradicional. Adquiere, en cambio, la forma de un relato" (Efland et al., 2003. p. 193)

En este caso, cuando cada profesora o profesor describe su práctica pedagógica vinculada a las artes visuales, señala diferentes experiencias, acciones y acontecimientos. Su relato nos expresa una verdad narrativa, la cual puede llegar a desarrollar una fuerza expresiva tal que nos lleva a dejar de lado la pregunta ¿será verdad? La verdad del sujeto está presente en su expresividad, en la forma en que articula los acontecimientos y en su capacidad de convencernos de que "así sucedieron las cosas".

Esta metodología se desarrolla en una conversación abierta, en la cual se produce aquello que muy bien señala Eisner (1998): la construcción de un texto narrativo se realiza de modo que pueda ser compartido por quienes estuvieron allí, permitiendo volver a vivenciar las situaciones desde los protagonistas. Así aparece la propia verdad narrativa del relato, queda en evidencia la experiencia como acontecimiento que emerge en el recuerdo de quien lo vive. Lo hace presente configurando su propio relato, mediante una verdad narrativa real e inmediata.

Así, la verdad narrativa posee una densidad que puede determinarse mediante la constitución de diferentes dominios de existencia, que requieren de enfoques disciplinares que pueden considerarse de un modo más amplio. Cuando Bertaux (2005) alude a una mirada etnográfica para constituir un relato de vida, señala que se puede distinguir entre distintas categorías y dimensiones. Bertaux (2005) invita a una mirada "etno-histórico-sociológica" (Bertaux, 2005), debido a que cierta época del trabajo sociológico se abstraía de que los fenómenos sociales se inscriben en un devenir histórico general de transformación social (Bertaux, 2005). Hoy, en general, podemos decir que las dinámicas de cambio social en diferentes dominios son parte de nuestros supuestos fundamentales para comprender procesos dados no solo alrededor del sujeto, sino también su impacto en él. Ello trae consigo que el cambio a nivel colectivo y su diversidad determinan efectos en la vida individual de las personas profesionales.

\section{Superando modos habituales de investigar en educación artística}

La narrativa permite superar los modos habituales como se realiza la investigación educativa, donde el objetivo principal es comprobar la teoría descubierta y luego intervenir, pero de algún modo "silenciando" las voces de las personas participantes. Durante el siglo XX, mucho se ha discutido acerca de la crisis que puede experimentar la racionalidad técnica, 
http://doi.org/10.15359/ree.24-3.29

http://www.una.ac.cr/educare

educare@una.ac.cr

ya que detrás de un pensamiento que da cuenta antes de los hechos concretos que de los efectos subjetivos que estos mismos generan, la narración permite abrir nuevas posibilidades de comprensión para captar la riqueza de las experiencias y develar el conocimiento que nace de la propia acción. Los relatos de vida acceden a la vida cotidiana, a las vivencias particulares en contextos institucionales determinados, captan la voz de los protagonistas y los convierten en el centro de la construcción y la reflexión teórica: una manera de construir una racionalidad que trasciende los efectos y se constituye como testimonio del ser subjetivo.

Sin embargo, considerar los relatos como un enfoque de investigación es tomar en cuenta una invitación a lo complejo con sus propios riesgos, especialmente cuando las miradas de lo artístico no siempre están en concordancia con el saber pedagógico que se inscribe en el trabajo artístico del aula. En el ámbito de la educación artística, la comprensión de los contextos locales y su implicancia en el currículo se puede profundizar al tomar en cuenta los relatos múltiples tanto de estudiantes como de profesoras y profesores, casi como un enfoque antropológico. Al mismo tiempo, esta comprensión permitiría obtener una mayor diversidad cultural al momento de pensar el curriculo (Efland et al., 2003), lo cual incluye tanto el contexto como la voz de quienes operan en él.

En este sentido, durante las últimas décadas, el campo de investigación en artes visuales y su reflexión en torno a lo educativo se ha abierto a metodologías que validan la voz propia como sitio de enunciación posible. Es el caso, por ejemplo, de la autoetnografía, que permite tomar en cuenta una perspectiva que no proviene "desde fuera", sino que toma en cuenta la voz de la persona que está involucrada en el contexto (Adams y Holman Jones, 2019). Lo anterior es significativo, pues el relato biográfico posee una apropiación de sentido que los estudios convencionales, situados la mayoría de las veces desde el punto de vista de un sujeto observador externo (en una posición de distancia que ha caracterizado tradicionalmente a la teoría) no son siempre capaces de articular completamente el sentido de lo que acontece desde los actores involucrados, donde se rescate la colaboración y el diálogo como un espacio de reflexión y construcción conceptual.

Por este motivo, creemos que resulta fundamental la necesidad de levantar líneas de investigación que tomen en cuenta la acción docente y la relación que sostiene con prácticas pedagógicas situadas en la experiencia contextualizada, con el fin de establecer conexiones con la cultura, y que se dan muchas veces en el espacio interdisciplinar. La investigación en el área de la educación artística vinculada a la experiencia vital docente es aún una tarea pendiente. Esta experiencia vital posee diferentes dimensiones, que van desde la formación escolar, las motivaciones vocacionales, los contextos familiares, los episodios que guiaron al profesorado de artes visuales a seguir el camino docente en sintonía con la exploración artística, hasta el azar y la conciencia profesional por citar solo algunas. 
Los países iberoamericanos necesitan impulsar la investigación en educación artística, sistematizar experiencias, desarrollar y compartir metodologías y didácticas, elementos que sin duda constituyen campos abiertos, de cuyo avance depende la posibilidad de la comprensión, sistematización y proyección de sus posibilidades dentro de los sistemas educativos en los niveles básico, medio superior y superior. (Jiménez et al., 2009, p. 12)

Esto constituye una oportunidad de propiciar la reflexión ligada "a la creación artística $y$, por sobre todo, [una manera de comprender] la sensibilidad como un pensamiento que sobrepasa el análisis racional y la habilidad [localizada en] un manejo instrumental de la representación" (Raquimán Ortega y Zamorano Sanhueza, 2017, p. 441). Lo anterior conlleva a pensar reflexivamente la idea que las profesoras y los profesores tienen con respecto a su definición de lo artístico. Con esto se propicia una mayor densidad discursiva y analítica de las artes visuales en el aula, sobrepasando el formalismo de la imagen (Raquimán Ortega y Zamorano Sanhueza, 2017). De este modo, al considerar temáticas emergentes en torno al mundo circundante, sería más factible considerar el arte como una expresión de vida tanto de quienes producen arte, como de aquellos entes que facilitan la detonación de procesos. También, propiciar el reconocimiento a una mirada más diversa, dando la oportunidad a manifestaciones artísticas locales y a los pequeños relatos que las constituyen. En este contexto, el arte cumple una función de construir la realidad (Efland et al., 2003) y, por este motivo, es de suma importancia valorar las construcciones que se dan en el contexto próximo del alumnado, como una posibilidad de reflexión y construcción situada en su propio entorno vital.

La identificación del arte como producción construida desde la cultura, la superación del concepto de progreso lineal y temporal, el reconocimiento de la diferencia, la democratización de las artes, la polisemia y la comprensión del contexto constituyen características sobresalientes dentro del enfoque epistemológico posmoderno (Efland et. al., 2003). Esto ha generado la necesidad de volver a pensar en las formas de concebir la labor docente en cuanto a sus modos de abordar la complejidad de la producción artística visual de hoy y el contexto en que se sitúan sus estudiantes. La reflexión que se puede generar es privilegiada, pues se trata de un encuentro del profesorado con su práctica pedagógica cotidiana. Considerando lo anterior, el proceso de enseñanza y aprendizaje se encuentra expuesto a tensiones que, justamente, intensifican el reconocimiento de lo complejo, de lo ambiguo y de la alteridad propiciada por los relatos deslocalizados de una historia hegemónica. La complejidad señalada no alude, necesariamente, a una dificultad que tiene que ver con aspectos puntuales de la creación artística o a sus modos más adecuados para estimular los aprendizajes en diferentes contextos educativos. Se trata más bien de una complejidad definida como un entramado de relaciones entre diversas disciplinas que, cada vez más, aparecen en concordancia con los procesos de producción; que requiere un modo de ser procesada y administrada de acuerdo con la pertinencia del relato de sus participantes. 
http://doi.org/10.15359/ree.24-3.29

http://www.una.ac.cr/educare

educare@una.ac.cr

Considerando este estado de cosas, las percepciones subjetivas adquieren una relevancia sustantiva para la investigación en educación artística. La narración otorga, al sujeto investigador en el campo educativo, una oportunidad excepcional para el diálogo intersubjetivo, en un intento de llegar a develar el significado del relato profesional docente, partiendo de la base de que esta "autoescritura" será resultado de una articulación dada entre el yo narrado, el sujeto narrador y el locutario (sujeto receptor del relato).

No se debe olvidar que el sujeto que narra, además de poder hablar y actuar, tiene la capacidad de unificar su vida (en este caso profesional) en la historia que cuenta, de la que se reconoce como personaje protagonista. Por esto, develar la transformación del discurso en un acontecimiento que condense su propia visión profesional y personal es reconocer que el discurso no solo acontece como descriptor, sino que también significa como conciencia. El esfuerzo, a partir de esto, es comprender el discurso en su sentido final, cuando se convierte en una constatación acerca de cómo ciertos fenómenos vitales confluyen en la propia práctica profesional.

Las narrativas docentes se construyen para comprender una determinada realidad social. Se concibe, en este contexto, la narrativa como "la cualidad estructurada de la experiencia entendida y vista como un relato; por [otra parte] ..., las pautas y formas de construir sentido, a partir de acciones temporales personales, por medio de la descripción y análisis de los datos" (Bolívar Botía, 2002, p. 5). En este ámbito, la narración permite comprender la complejidad que poseen las experiencias de los individuos, sus conflictos y dilemas profesionales, "es muy probable que la importancia de la narración para la cohesión de una cultura sea tan grande como lo es para la estructuración de la vida de un individuo" (Brunner, 2000, p. 59).

Las instituciones y las prácticas sociales tienen historias, y la comprensión de esas prácticas se asume con frecuencia como un relato. A través de este mismo, las personas profesionales reconocen que las prácticas cambian con el tiempo. Se caracterizan por construirse en el pasado y los esfuerzos de entender los testimonios sobre las prácticas pedagógicas permiten definir los propósitos que ha tenido el profesorado para actuar, determinar cómo lo han hecho, qué decisiones han tomado y en qué circunstancias.

En este contexto, las voces del profesorado, que relatan sus prácticas como posibilidad de entender la realidad, generan la construcción de un modo de comprensión y expresión de la vida profesional, en la cual se hace presente su voz.

Estos relatos proporcionan la oportunidad de que sean las personas profesionales mismas quienes expresen "cómo definen y comprenden sus propias vidas. Rescatar la dimensión personal del oficio de enseñar es, también, un modo de oponerse al profesorado anónimo, sin nombre e impersonal" (Bolívar Botía et al., 2001, p. 52). La narrativa permite que se destaque el carácter complejo e interrelacionado de los fenómenos vitales, considerando que este tipo de investigaciones poseen un proceso en que continuamente se intenta dar cuenta de los diferentes niveles, tanto temporales como sociales, que se van dando en el transcurso del estudio. 


\section{Hacia una conclusión}

Los marcos conceptuales que han influido sobre las prácticas de educación artística "en las últimas décadas han adquirido una mayor densidad discursiva, lo cual constituye un potencial soporte para generar acercamientos relevantes" (Raquimán Ortega y Zamorano Sanhueza, 2017, p. 440) en lo que se refiere a la comprensión de cómo se construye la realidad en el cotidiano de la sala de clases. La exposición de problemáticas contingentes es parte de un potencial imaginario colectivo que incide cada vez más en la educación artística. Por ejemplo, para Dobbs (2004), la condición humana y la experiencia parecen determinar nuevas interrogantes en relación con las interpretaciones que pueden darse en el terreno de lo educativo. En la medida en que la cultura se vincula con fenómenos educacionales, resulta consistente preguntarse de qué manera el conocimiento local proporciona puntos de vista diversos, cuya característica central es la subjetividad que se inscribe en el ámbito del aula. La ya clásica definición de cultura de Geertz (2003) es útil para comprender de qué manera la interpretación posee un papel central y el concepto de cultura en ella:

Es esencialmente un concepto semiótico. Creyendo con Max Weber que el hombre es un animal inserto en tramas de significación que él mismo ha tejido, considero que la cultura es esa urdimbre y que el análisis de la cultura ha de ser por tanto, no una ciencia experimental en busca de leyes, sino una ciencia interpretativa en busca de significaciones. Lo que busco es la explicación, interpretando expresiones sociales que son enigmáticas en su superficie. (Geertz, 2003, p. 20)

La indagación cualitativa se vincula, entonces, con el fenómeno de la producción artística, que se enlaza, a su vez, con las prácticas educacionales que la toman como su objeto de estudio, conocimiento y experiencia: "la investigación comprende, pues, todo intento sistemático por comprender, en profundidad, el modo en que se enseña y se aprende el arte" (Torres de Eça y Agra Pardiñas, 2016, p. 20). Se trata de pensar en la producción artística visual como un conjunto de procesos intelectuales, sensibles y conceptuales que dan cuenta de la experiencia individual situada en un marco de relaciones complejas, que son dependientes de la biografía de cada estudiante.

Debido a lo anterior, la educación artística que se vincula con una mirada desde el contexto también ha tenido que abordar estrategias de enseñanza que consideren las motivaciones, alcances y límites de la experiencia del arte localizado en el espacio escolar cotidiano. Este espacio se convierte en una potencial riqueza de la comprensión del fenómeno artístico como un espacio de manifestaciones culturales propias: "estudiar una forma de arte significa explorar una sensibilidad, que una sensibilidad semejante es esencialmente una formación colectiva y que los fundamentos de esa formación son tan amplios y profundos como la existencia social" (Geertz, 1994, p. 112). La comprensión de la sensibilidad en un nivel más subjetivo puede 
http://doi.org/10.15359/ree.24-3.29

http://www.una.ac.cr/educare

educare@una.ac.cr

propiciar, también, preguntas en torno a las condiciones vitales más personales de quienes operan en el dominio de la enseñanza artística. En este sentido, las autobiografías representan formas de articular discursos que den cuenta de cómo se constituye la acción docente en relación con la experiencia individual y el contexto social.

Las historias de vida permiten identificar y contextualizar posibles representaciones o modelos sobre los propios posicionamientos que tiene el profesorado en relación con componentes éticos, sociales y culturales. Este aspecto se transforma en una oportunidad para hacer emerger nuevas aproximaciones a las problemáticas que se vislumbran día a día en las aulas, donde los relatos son una oportunidad de prestar atención a lo singular y hacer emerger aspectos de la propia vida que permiten reconstruir el propio proyecto de vida.

La reconstrucción de la experiencia cotidiana y la reflexión de cómo se desenvuelven y consideran aspectos de la cultura local entregan una posibilidad de comprensión y transformación social, que pretende relevar al profesorado y a los diferentes espacios donde se desempeña. Al investigar se proponen nuevos puntos de vistas, no se persigue establecer dogmas, ya que estamos en una época en que la incertidumbre es la protagonista: "se trata de identificar cuestiones y problemas, de recoger y registrar informaciones, de diseñar nuevos modelos que enriquezcan el conocimiento que tenemos de las cosas" (Torres de Eça y Agra Pardiñas, 2016, p. 20). Junto a ello, esta opción investigativa se configura como un aporte a la formación inicial del profesorado de artes visuales, particularmente, en el sentido de promover experiencias que favorezcan, en los profesores y las profesoras en formación, la incorporación del contexto local, sus características y fortalezas junto a todo su potencial transformador, "no solo porque la exposición de las historias tiene consecuencias sobre nuestras vidas y las vidas de los demás, sino también debido a los procesos de localización, contextualización, problematización y experimentación que emergen del ejercio de escritura de un relato autobiografico" (Hernández Hernández y Rifà Valls, 2011, p. 45), con el fin de resituar el yo y la identidad en un espacio reflexivo que permita reconocer historias personales y el entretejido entre los relatos y sus posibles referentes teóricos.

\section{Referencias}

AcevesLozano,J.E.(1999). Un enfoquemetodológico delas historias devida.Proposiciones, 29, 45-51. https://www.sitiosur.cl/?s=Un+enfoque+metodol\%C3\%B3gico+de+las+historias+de+vida

Adams, T. E. y Holman Jones, S. (2019). The art of autoethnography. En P. Leavy (Ed.), Handbook of arts-based research (pp. 141-164). Guilford Publications.

Agra Pardiñas, M. X. (2006). El vuelo de la mariposa: La investigación artístico-narrativa como herramienta de formación. En R. Marín Viadel (Ed.), Investigación en educación artística (pp. 127-150). Editorial Universidad de Granada. 
http://doi.org/10.15359/ree.24-3.29

Albero, B., Linard, M. y Robin, J.-Y. (2008). Petite fabrique de l'innovation à l'université. Quatre parcours de pionniers. L'Harmattan.

Bertaux, D. (1997). Les récits de vie. Perspective ethnosociologique. Nathan.

Bertaux, D. (2005). Los relatos de vida. Perspectiva etnosociológica. Ediciones Bellaterra.

Blumer, H. (1998). Symbolic interactionism: Perspective and method. University of California.

Bolívar Botía, A. (2002). ¿De nobis ipsis silemus?: Epistemología de la investigación biográficonarrativa en educación. Revista Electrónica de Investigación Educativa, 4(1), 1-26. https:// redie.uabc.mx/redie/article/viewFile/49/91

Bolívar Botía, A., Domingo Segovia, J. y Fernández Cruz, M. (1998). La investigación biográficonarrativa en educación. Guía para indagar en el campo. FORCE; Universidad de Granada y Grupo Editorial Universitario.

Bolívar Botía, A., Domingo Segovia, J. y Fernández Cruz, M. (2001). La investigación biográficonarrativa en educación. Enfoque y metodología. Editorial La Muralla.

Bourdieu, E. (Dirección). (1999). La miseria del mundo. Fondo de Cultura Económica.

Bruner, J. (2000). La educación, puerta de la cultura. Antonio Machado Libros

Corcuff, P. (1998). Las nuevas sociologías. Principales corrientes y debates, 1980-2010. Alianza Editorial.

de Villers, G. (1996). L'approche biographique au carrefour de la formation des adultes, de la recherche et de l'intervention. Le récit de vie comme approche de recherche-formation. En D. Desmarais y J.-M. Pilon (Coords.), Pratiques des histoires de vie. Au carrefour de la formation, de la recherche et de l'intervention (pp. 107-134). L'Harmattan.

Dobbs, S. M. (2004). Discipline-based art education. En E. W. Eisner y M. D. Day (Eds.), Handbook of research and policy in art education (pp. 701-724). Lawrence Erlbaum Associates. https:// doi.org/10.4324/9781410609939

Efland, A. D., Freedman, K. y Stuhr, P. (2003). La educación en el arte posmoderno. Paidós.

Eisner, E. W. (1998). El ojo ilustrado. Indagación cualitativa y mejora de la práctica educativa. Paidós.

Freire Hermosilla, A., Retamal Salazar, J. y Sepúlveda Leiva, R. (2005). La aventura epistemológica de nuestra propia comprensión. Una mirada desde la pedagogía. Ediciones UCSH.

Geertz, C. (1994). Conocimiento local: Ensayos sobre la interpretación de las culturas. Paidós.

Geertz, C. (2003). La interpretación de las culturas. Gedisa. 
http://doi.org/10.15359/ree.24-3.29

http://www.una.ac.cr/educare

educare@una.ac.cr

Giannini, H. (2004). La "reflexión" cotidiana. Hacia una arqueología de la experiencia. Editorial Universitaria.

Hernández Hernández, F.y Rifà Valls, M. (2011). Para una génesis de la investigación autobiográfica y de su lugar en educación. En F. Hernández Hernández y M. Rifà Valls (Coods.), Investigación autobiográfica y cambio social (pp. 21-48). Octaedro.

Jiménez, L., Aguirre, I. y Pimentel, L. G. (2009). Educación artística, cultura y ciudadanía. Fundación Santillana. https://www.academia.edu/11763957/Educaci\%C3\%B3n art\%C3\%ADstica cultura y ciudadan\%C3\%ADa

Kornblit, A. L. (2004). Historias y relatos de vida: Una herramienta clave en metodologías cualitativas. En A. L. Kornblit (Coord.), Metodologías cualitativas en ciencias sociales. Modelos y procedimientos de análisis (pp. 15-27). Biblos.

Lewis, O. (1964). Los hijos de Sánchez. Joaquín Mortiz

Luque Ramos, P. J. y Cruz Elvira, C. M. (2016). 2. Prácticas cualitativas para la investigación sobre las artes. En M. I. Moreno Montoro y M. P. López-Peláez Casellas (Coords.), Reflexiones sobre investigación artística e investigación educativa basada en las artes (pp. 43-58). Sintesis.

Martuccelli, D. y de Singly, F. (2012). Las sociologías del individuo. LOM.

Mella Valenzuela, O. (2003). Metodología cualitativa en ciencias sociales y educación. Primus.

Raquimán Ortega, P. y Zamorano Sanhueza, M. (2017). Didáctica de las artes visuales, una aproximación desde sus enfoques de enseñanza. Estudios pedagógicos, 43(1), 439-456. https://dx.doi.org/10.4067/S0718-07052017000100025

Schwartz, H. y Jacobs, J. (1984). Sociología cualitativa. Método para la reconstrucción de la realidad. Trillas.

Thomas, W. I. y Znaniecki, F. (2004). El campesino polaco en Europa y en América. CIS; BOE.

Torres de Eça, T. y Agra Pardiñas, M. J. (2016). Prólogo. Como pretexto para pensar en voz alta. En M. I. Moreno Montoro y M. P. López-Peláez Casellas (Coords.), Reflexiones sobre investigación artística e investigación educativa basada en las artes (pp. 19-26). Sintesis.

Weber, M. (2001). Ensayos sobre metodología sociológica. Amorrortu. 